\title{
Article \\ Identification of Three Novel PmGRI1 Genomic Resistance Islands and One Multidrug Resistant Hybrid Structure of Tn7-like Transposon and PmGRI1 in Proteus mirabilis
}

\author{
Boheng Ma ${ }^{1,2,3,+}$, Xuechun Wang ${ }^{1,2,3,+}$, Changwei Lei ${ }^{1,2,3} \mathbb{D}$, Yizhi Tang ${ }^{1,2,3}$, Juan He ${ }^{1,2,3}$, Yufeng Gao ${ }^{1,2,3}$, \\ Yu Zhang ${ }^{1,2,3}$ and Hongning Wang ${ }^{1,2,3, *}$ \\ 1 College of Life Sciences, Sichuan University, Chengdu 610065, China; bohengm@163.com (B.M.); \\ wangxuechuns@126.com (X.W.); leichangwei@126.com (C.L.); tangyizhi23@163.com (Y.T.); \\ 2018222040040@stu.scu.edu.cn (J.H.); 2019222040103@stu.scu.edu.cn (Y.G.); yuzhang712@163.com (Y.Z.) \\ 2 Key Laboratory of Bio-Resource and Eco-Environment of Ministry of Education, Sichuan University, \\ Chengdu 610065, China \\ 3 Animal Disease Prevention and Food Safety Key Laboratory of Sichuan Province, Sichuan University, \\ Chengdu 610065, China \\ * Correspondence: whongning@163.com \\ + Boheng Ma and Xuechun Wang contributed equally to this work and share first authorship.
}

Citation: Ma, B.; Wang, X.; Lei, C.; Tang, Y.; He, J.; Gao, Y.; Zhang, Y.; Wang, H. Identification of Three Novel PmGRI1 Genomic Resistance Islands and One Multidrug Resistant Hybrid Structure of Tn7-like Transposon and PmGRI1 in Proteus mirabilis. Antibiotics 2021, 10, 1268. https://doi.org/10.3390/antibiotics 10101268

Academic Editors: Laila Ben Said, Ismail Fliss and Carmen Torres

Received: 23 September 2021 Accepted: 16 October 2021

Published: 18 October 2021

Publisher's Note: MDPI stays neutral with regard to jurisdictional claims in published maps and institutional affiliations.

Copyright: (c) 2021 by the authors. Licensee MDPI, Basel, Switzerland. This article is an open access article distributed under the terms and conditions of the Creative Commons Attribution (CC BY) license (https:/ / creativecommons.org/licenses/by/ $4.0 /)$.

\begin{abstract}
The widespread use of antibiotics in large-scale livestock production has led to serious antibiotic resistance. Proteus mirabilis is an important pathogenic bacterium on large-scale farms. Chromosomally localized mobilizable genetic elements (genomic islands) and mobile genetic elements (Tn7-like transposons) play an important role in the acquisition and transmission of resistance genes by $P$. mirabilis. To study the prevalence and resistance characteristics of antibiotic-resistant genomic islands in P. mirabilis of animal origin in China, we performed whole genome sequencing of P. mirabilis isolated from large-scale pig and chicken farms. Three new variants of PmGRI1 (HN31, YN8, and YN9), and a hybrid structure (HN2p) formed by the multidrug-resistant Tn7-like-HN2p transposon and a genomic island PmGRI1-HN2p, were identified from P. mirabilis. All variants underwent homologous recombination mediated by insertion sequence IS26. A genomic rearrangement in the chromosome between the Tn7-like-HN2p transposon and PmGRI1-HN2p occurred in HN2p. The heterozygous structure contained various antimicrobial resistance genes, including three copies of fluoroquinolone resistance gene $q n r A 1$ and $16 \mathrm{~S}$ rRNA methylase gene $r m t B$, which are rarely found in P. mirabilis. Our results highlight the structural genetic diversity of genomic islands by characterizing the novel variants of PmGRI1 and enrich the research base of multidrug resistance genomic islands.
\end{abstract}

Keywords: Proteus mirabilis; antibiotic resistance; genomic island; PmGRI1; Tn7

\section{Introduction}

Proteus mirabilis belongs to the Enterobacteriaceae family and is widely distributed in the environment and the intestinal tract of living organisms. It can carry numerous pathogenic factors that may be associated with gastrointestinal pathogenicity [1,2]. P. mirabilis is inherently resistant to antibiotics, such as nitrofurantoin, polymyxin, and tigecycline [3], often exhibiting multidrug resistance under clinical settings. Previous studies have reported multidrug resistance rates of $19.3-78.13 \%$ in P. mirabilis isolated from poultry [4-6]. Mobilizable genetic elements (genomic islands (GIs) and Tn7-like transposons) play important roles in the capture and transmission of multidrug resistance genes in P. mirabilis [7-10]. GIs, such as integrative and conjugative elements and integrative and mobilizable elements, can integrate gene fragments or single-stranded DNA into bacterial chromosomes via horizontal gene transfer and often contain various genes that confer novel traits to their hosts, such as antibiotic resistance, virulence, and enhanced adaptation of bacteria to their environment [11-13]. Recently, traces of GIs have been identified in an increasing 
number of host bacteria. Multidrug-resistant GIs carrying the macrolide resistance gene $\operatorname{erm}(\mathrm{B})$ have been reported in Campylobacter of animal origin, and erm(B) was found to be prevalent and significantly increased in Campylobacter in Guangdong Province, China. It is speculated that GIs may be widespread or play a role in the transmission of erm(B) [14]. A GI carrying a new variant of tet $(\mathrm{L})$ was identified in Campylobacter of chicken origin; the variant was found to play an important role in tetracycline and doxycycline antibiotic resistance and could make tigecycline less susceptible [15]. GIs associated with glycopeptides, chloramphenicol, aminoglycosides, tetracyclines, sulfonamides, and $\beta$-lactam antibiotic resistance were identified in Riemerella anatipestifer by pan-genomic analysis [16]. Several novel multidrug-resistant GIs have been identified in Trueperella pyogenes of porcine origin, carrying multidrug resistance genes, such as the tetracycline resistance gene tet $(\mathrm{W})$ and the macrolide resistance gene $\mathrm{erm}(\mathrm{X})$ [17]. Previous studies have reported that the most common genetic island in P. mirabilis is the Salmonella genomic island 1 (SGI1) [18-20] and the Proteus genomic island 1 (PGI1; [21,22]). The host bacteria range of SGI1 is very broad, and its presence in P. mirabilis, Morganella morganii, Providencia stuartii, and Escherichia coli, as reported in previous studies, suggests that SGI1 could spread to Enterobacteriaceae [18,23-25].

It has been reported that novel multidrug-resistant GIs, including PGI1, PGI2, and GIPmI1, were found in P. mirabilis $[7,21,26]$. PmGRI1 is a newly reported GI with variants ranging in size from 26,073 to $150,977 \mathrm{bp}$ that can carry multiple resistance genes and has been identified in P. mirabilis and E. coli. It carries a tyrosine-type recombinant/integrase (394 amino acids) and is predicted to catalyze the integration of PmGRI1 at the $3^{\prime}$ end of tRNA(Sec). Different PmGRI1 variants with backbone alterations and/or variants in the MDR region carrying the carbapenemase gene $b l a_{\mathrm{KPC}-2}$ and the $16 \mathrm{~S}$ rRNA methylesterase gene arm $A$ have been identified in P. mirabilis, suggesting that PmGRI1 can carry clinically important resistance genes [27].

The Tn7-like transposon can transfer resistance genes $[10,28]$. Sequences at both ends of the Tn7-like transposon encode transposon modules and class 2 integrator systems. The transposon module encodes five proteins required for both transposition pathways: TnsA, TnsB, TnsC, TnsD, and TnsE [29]. Although these gene cassettes are fixed in Tn7 transposons due to mutations in homologous recombinases, they can be rearranged in hosts expressing related recombinases, leading to alternative combinations of antibiotic resistance genes. Tn7-like transposons can promote the spread of resistance levels in bacteria by transferring various resistance genes between bacteria via transposases. Furthermore, a Tn7-like transposon, Tn6450, contains 18 different antimicrobial resistance genes, including the cephalosporinase $b l a_{\mathrm{DHA}-1}$ and the fluoroquinolone resistance genes $q n r A 1$ and $a a c\left(6^{\prime}\right)$ Ib-cr [10].

\section{Materials and Methods}

\subsection{Bacterial Strains}

In 2019, 204 strains of Proteus mirabilis were isolated from the feces of large pig and chicken farms in Henan and Yunnan Provinces, China. The strains were cultured using Salmonella-Shigella agar (SS) at $37^{\circ} \mathrm{C}$ for $16 \mathrm{~h}$. The strains were identified using the BD Phoenix ${ }^{\mathrm{TM}} 100$ Automated Microbiology System (Becton Dickinson, Franklin Lakes, NJ, USA).

\subsection{Antimicrobial Susceptibility Testing}

Antibiotic susceptibility testing of the strains was performed using the K-B diskdiffusion method. The 27th edition of the Executive Standard for Antimicrobial Susceptibility Testing (M100-S27), prepared by the Clinical and Laboratory Standards Institute, was used as a reference (Table S1). Drug sensitivity testing was carried out using Oxoid Paper Dispenser Type ST6090 and the OXOID drug sensitivity disks (OXOID, Basingstoke, UK). The quality control strain for the drug sensitivity test was E. coli ATCC25922. A total of 19 antibiotics were used for Antibiotic susceptibility testing. The antibiotics included ceftazidime (CAZ), aztreonam (ATM), levofloxacin (LEV), ampicillin (AMP), amoxicillin-clavulanic 
acid (AMC), cefoxitin (FOX), cefotaxime (CTX), chloramphenicol (CHL), imipenem (IPM), florfenicol (FFC), nalidixic acid (NAL), ciprofloxacin (CIP), streptomycin (STR), spectacularin (SPT), gentamicin (GEN), amikacin (AMK), methicillin (TMP), sulforaphane (SUL), and trimethoprim-sulfamethoxazole (SXT).

\subsection{DNA Extraction}

Bacterial genomic DNA was extracted using the QIAamp DNA Mini Stool kit (QIAamp, Hilden, Germany) according to the manufacturer's instructions, and the genomic DNA concentration and quality were checked using a NanoDrop spectrophotometer and via agarose gel electrophoresis. The obtained DNA was stored at $-20{ }^{\circ} \mathrm{C}$ until further analysis.

\subsection{Whole Genome Sequencing and Analysis}

Whole genome sequencing of 7 strains of multidrug-resistant $P$. mirabilis was selected from 204 strains of $P$. mirabilis. The whole genome of all strains was sequenced using the Illumina HiSeq platform (San Diego, CA, USA) (400 bp paired-end reads with about 200-fold average coverage) and a Nanopore sequencing instrument (MinION, Oxford, UK) (about 400-fold average read depth). After DNA extraction, $1 \mu$ g genomic DNA was randomly fragmented by Covaris (Woburn, MA, USA), followed by purification by an AxyPrep Mag PCR clean-up kit (Union City, CA, USA). The fragments were end-repaired by End Repair Mix and purified afterward. The repaired DNAs were combined with A-Tailing Mix, and then the Illumina adaptors were ligated to the Adenylate $3^{\prime}$ Ends DNA and followed by product purification. The products were selected based on the insert size. Several rounds of PCR amplification with PCR Primer Cocktail and PCR Master Mix were performed to enrich the Adapter-ligated DNA fragments. After purification, the library was qualified by the Agilent Technologies 2100 (Palo Alto, CA, USA) bioanalyzer and ABI StepOnePlus Realtime PCR System (Foster City, CA, USA). Finally, the qualified libraries were sequenced pair-end using Hiseq System.

The raw sequencing data were processed using the following steps: (1) Removing reads containing sequencing adapter; (2) Removing reads whose low-quality base ratio (base quality less than or equal to 5) is more than 50\%; (3) Removing reads whose unknown base (' $\mathrm{N}$ ' base) ratio is more than $10 \%$. Clean data were aligned to the reference genome using Burrows-Wheeler Aligner (BWA) [30]. Picard was used to remove duplicated sequence reads. Realignment was performed with the Genome Analysis Toolkit (GATK) [31] Single-nucleotide polymorphisms (SNPs) and insertions-deletions (InDels) were called using HaplotypeCaller of GATK and annotated with SnpEff software [32]. The Copy Number Variants (CNVs) were called using the CNVnator read-depth algorithm [33]. CREST was used to identify structural variants (SVs) with standard settings [34].

The genomes were assembled using the Canu v1.5 software [35]. Antimicrobial resistance genes were identified using CGE ResFinder 3.1 (https:/ / cge.cbs.dtu.dk/services / ResFinder/, accessed on 8 October 2021) [36]. Insertion sequences were identified using ISfinder (https: / / www-is.biotoul.fr/, accessed on 8 October 2021) [37]. The plasmids were identified using PlasmidFinder 2.1 [38,39]. The complete nucleotide sequence of the hybrid structure was analyzed using the BLAST program (http:/ / blast.ncbi.nlm.nih.gov/Blast.cgi, accessed on 8 October 2021).

\section{Results and Discussion}

\subsection{Antibiotic Susceptibility and Detection of Antimicrobial Resistance Genes}

In addition to intrinsic resistance to doxycycline and polymyxin, $P$. mirabilis strains HN31, YN8, YN9, and HN2p are all insensitive to a variety of antibiotics and carry multiple drug resistance genes (Table 1). In addition, they all carry the merEDACPTR mercuryresistance operon. Our results highlight that PmGRI1 and the hybrid structure of Tn7-like and PmGRI1 can carry numerous antibiotic resistance genes, which may be one of the important reasons for the acquisition and spread of antibiotic resistance genes in P. mirabilis. The PlasmidFinder results showed that YN8 did not carry a plasmid and that HN31, YN9, 
and HN2P all carried IncQ1 plasmids but did not carry resistance genes on the plasmids.

The resistance genes were relatively concentrated on PmGRI1.

Table 1. Resistance genes and profile of PmGRI1 in P. mirabilis.

\begin{tabular}{|c|c|c|c|c|c|}
\hline Strain & $\begin{array}{c}\text { Source of } \\
\text { Samples }\end{array}$ & $\begin{array}{l}\text { SGI1/SGI1-like } \\
\text { Gene Island Type }\end{array}$ & Antibiotic Resistance Genes ${ }^{\text {a }}$ & $\begin{array}{l}\text { Strain Resistance } \\
\text { Profile }\end{array}$ & Size (bp) \\
\hline HN31 & Swine & PmGRI1-HN31 & $\begin{array}{c}\text { catA1, dfrA12, aadA2, sul1, mph(A), aacC2d, } \\
\text { sul2, strA, strB, aphA1, floR, tet }(A)\end{array}$ & $\begin{array}{l}\text { AMP-CHL-NAL- } \\
\text { STR-SPT-GEN- } \\
\text { TMP-SUL-SXT }\end{array}$ & 62,862 \\
\hline YN8 & Chicken & PmGRI1-YN8 & $\begin{array}{c}\text { catA1, bla } \text { CTX-M-65, fosA3, sul1, aadA5, }_{\text {dfrA17, aadA1, dfrA1, sul2 }} \\
\text { df }\end{array}$ & $\begin{array}{l}\text { NAL-SPT-TMP- } \\
\text { SUL-SXT }\end{array}$ & 62,784 \\
\hline YN9 & Chicken & PmGRI1-YN9 & 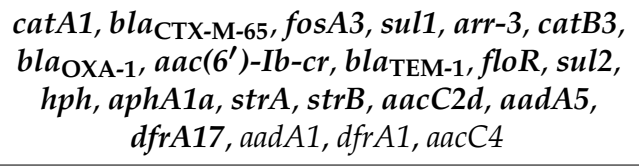 & $\begin{array}{l}\text { AMP-CHL-FFC- } \\
\text { NAL-CIP-STR- } \\
\text { SPT-GEN-TMP- } \\
\quad \text { SUL-SXT }\end{array}$ & 55,239 \\
\hline HN2p & Swine & $\begin{array}{l}\text { Hybrid structure } \\
\text { of Tn7-like and } \\
\text { PmGRI1 }\end{array}$ & 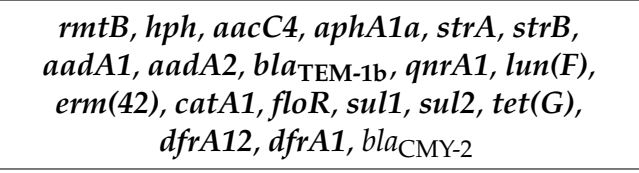 & $\begin{array}{l}\text { AMP-AMC-AMK- } \\
\text { SXT-LEV-CIP- } \\
\text { NAL-FFC-CHL- } \\
\text { GEN }\end{array}$ & 123,622 \\
\hline HN31 & Swine & PmGRI1-HN31 & $\begin{array}{c}\text { catA1, dfrA12, aadA2, sul1, mph }(A), \text { aacC2d, } \\
\text { sul2, strA, strB, aphA1, floR, tet }(A)\end{array}$ & $\begin{array}{l}\text { AMP-CHL-NAL- } \\
\text { STR-SPT-GEN- } \\
\text { TMP-SUL-SXT }\end{array}$ & 62,862 \\
\hline YN8 & Chicken & PmGRI1-YN8 & $\begin{array}{c}\text { catA1, } \text { bla }_{\mathrm{CTX}-\mathrm{M}-65}, \text { fosA3, sul1, aadA5, } \\
\text { dfrA17, aadA1, dfrA1, sul2 }\end{array}$ & $\begin{array}{l}\text { NAL-SPT-TMP- } \\
\text { SUL-SXT }\end{array}$ & 62,784 \\
\hline YN9 & Chicken & PmGRI1-YN9 & 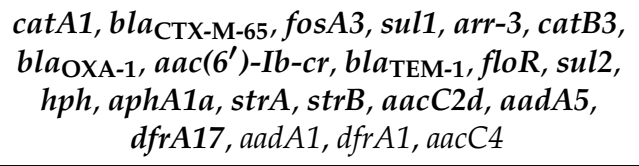 & $\begin{array}{l}\text { AMP-CHL-FFC- } \\
\text { NAL-CIP-STR- } \\
\text { SPT-GEN-TMP- } \\
\text { SUL-SXT }\end{array}$ & 55,239 \\
\hline HN2p & Swine & $\begin{array}{l}\text { Hybrid structure } \\
\text { of Tn7-like and } \\
\text { PmGRI1 }\end{array}$ & 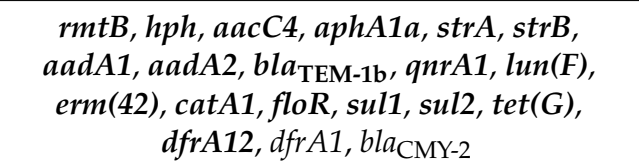 & $\begin{array}{l}\text { AMP-AMC-AMK- } \\
\text { SXT-LEV-CIP- } \\
\text { NAL-FFC-CHL- } \\
\text { GEN }\end{array}$ & 123,622 \\
\hline
\end{tabular}

a Antimicrobial resistance genes carried by PmGRI1 or hybrid structure are indicated in bold.

\subsection{Analysis of Three Novel Variants of PmGRI1 in P. mirabilis}

The whole genomes of strains HN31, YN8, and YN9 were compared by a BLAST search through NCBI, and all carried novel variants of PmGRI1 (PmGRI1-HN31, PmGRI1-YN8, and PmGRI1-YN9). The three novel variants of PmGRI1 were consistent with PmGRI1C55 (P. mirabilis strain C55 was isolated from cloacal swabs of a chicken with diarrhea in Shandong Province, China, on 13 November 2018; NCBI GenBank MK861851.1, [27]) and were integrated downstream of $\mathrm{tRNA}(\mathrm{Sec})$ with $20 \mathrm{bp}$ direct repeats (DR) at the left and right ends. They all underwent homologous recombination mediated by the insertion sequence IS26, due to which fragments from different plasmids or chromosomes were inserted, conferring new antibiotic resistance genes to the strain (Figure 1).

The size of PmGRI1-HN31 was $62,862 \mathrm{bp}$. The region downstream of Tn21 tnpA is similar to the fragment from Klebsiella pneumoniae plasmid pDA33144-220, both containing the resistance genes $d f r A 12$, aadA2, sul1, and $m p h(\mathrm{~A})$. This result suggests that this region may have been derived from pDA33144-220. Subsequently, insertions and reversals occur in the region between four IS26; an additional insertion of the aminoglycoside resistance gene $a a c C 2 d$ is upstream IS26, and the adjacent IS26-bla $a_{\mathrm{TEM}-1 \mathrm{~b}}-\mathrm{IS} 26-a p h A 1 a$ was reversed and transferred to a downstream IS26. However, IS26 and $b l a_{\mathrm{TEM}-1 \mathrm{~b}}$ were lost in PmGRI1-HN31 compared to those in PmGRI1-C55. 




Figure 1. Genetic structure of three novel variants of PmGRI1. Structures were drawn to scale from NCBI accession numbers MW699442 (PmGRI1-HN31), MW699444 (PmGRI1-YN8), MW699445(PmGRI1-YN9), MK861851 (PmGRI1-C55), CP029591 (pDA33144-220), CP053898 (P. mirabilis YPM35), and CP045257 (P. mirabilis L90-1). Genes and open reading frames are indicated by arrows, and arrowheads indicate their orientations of transcription. Shared regions are indicated by shading with $>99 \%$ identity. Antimicrobial resistance genes are shown in red, and transposase genes are shown in blue. The yellow arrow represents the integrase gene. The gray arrows represent other functional genes that have nothing to do with this research. The IS elements are indicated by boxes around the blue arrows. DR represents the $20 \mathrm{bp}$ direct repeats at the ends of PmGRI1.

PmGRI1-YN8 is 55,239 bp. The Tn21 tnpA of PmGRI1-YN8 was truncated by IS26, and a $14,852 \mathrm{bp}$ sequence was inserted between the two IS26. This sequence carries five antibiotic resistance genes- bla $_{\mathrm{CTX}-\mathrm{M}-65}$, fos $A 3$, sul1, aadA5, and $d f r A 17$.

PmGRI1-YN9 is $99,907 \mathrm{bp}$. It contains two sequences that are highly similar to P. mirabilis YPM35 and P. mirabilis L90-1 with a 99.9\% nucleotide similarity. These two sequences carry the mobile elements ISCR2 and ISVsa5. The sul1-qacE $\Delta 1$ gene cassette was observed on both ends of the inserted fragment, which may be associated with the insertion of a large fragment in this region. PmGRI1-YN9 contained 18 antibiotic resistance genes (Table 1).

IS26 moves through a replication mechanism and can be used to cause insertion or deletion, or can flip adjacent DNA, playing an important role in pathogen evolution [40-42]. In addition, translocatable units containing only an IS26 and a resistance gene are preferentially inserted into adjacent positions of the existing IS26 in the same cell, resulting in an IS26-bounded class 1 transposon [43]. Previous studies have reported that IS26mediated excision of the IS26-aphA1a gene transposon resulted in the loss of kanamycin resistance [44]. Our study showed that IS26-mediated homologous recombination of the backbone region and multidrug resistance region promoted the diversity of PmGRI1-like genomic island variants.

\subsection{Characteristics of the Hybrid Structure in P. mirabilis}

In the P. mirabilis strain HN2p, the SXT/R391 integrative and conjugative element ICEPmiJpn1 (GenBank accession KT894734), existing alone and harboring bla $a_{\mathrm{CMY}-2}$, was integrated with the end of $\operatorname{prfC}$. A resistant GI and a novel transposon derived from $\mathrm{Tn} 7$ exist together and undergo genome rearrangement between the two elements. 
The hybrid structure is formed by PmGRI1-HN2p and Tn7-like-HN2p (Figure 2). In HN2p, PmGRI1-HN2p is located between PMI3004 and PMI3005 compared with the P. mirabilis reference genome HI4320 (GenBank accession AM942759), and Tn7-like-HN2p is located at PMI3067. The two genetic elements have a Tn21 region, one of which is incomplete, likely due to the truncation of IS26 upstream. We hypothesized that an incomplete Tn21 transposon mediates the transfer of the gene fragment and divides the assemblies into two parts (corresponding to bases 1537 to 76,562 bp and 148,476 to 197,071 bp in GenBank accession number MT585156). This indicates that the Tn21 region might cause genome rearrangement. The region corresponding to PMI3005 to PMI3067 in P. mirabilis HI 4320 was reversed between the two parts, and the complete inverse part had a length of $145 \mathrm{~kb}$.

(a)

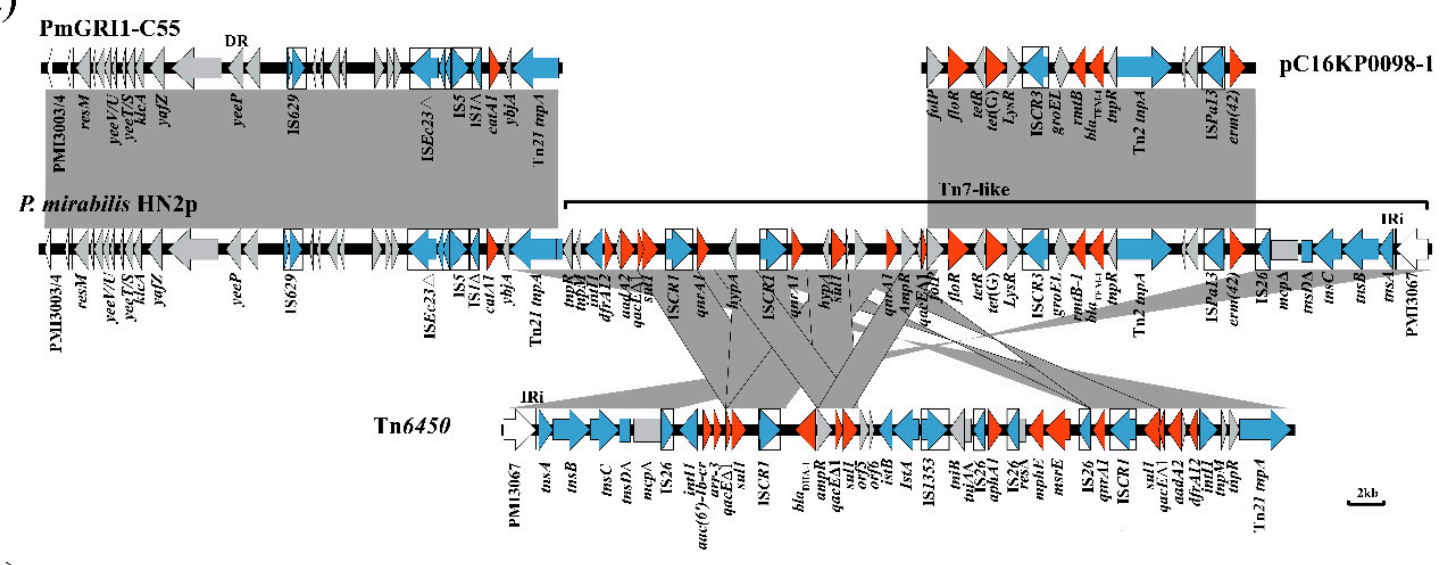

(b)

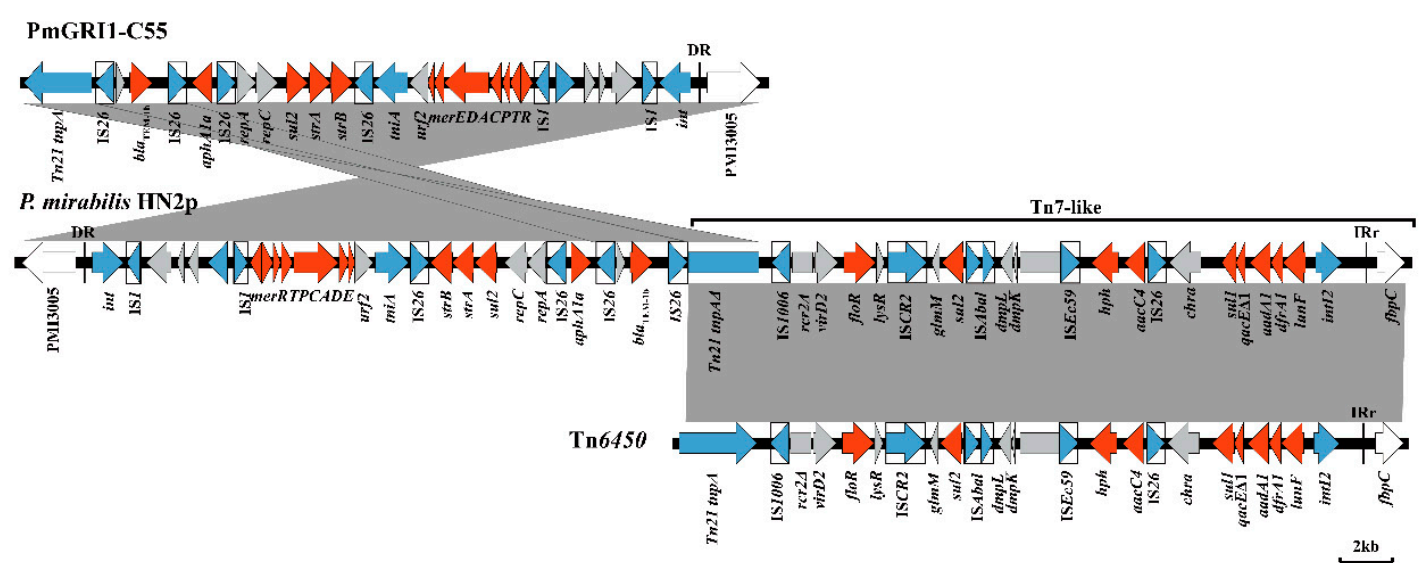

Figure 2. Genetic structures of the hybrid structure: (a) front end and (b) back end. Structures are drawn to scale from NCBI accession numbers MT585156 (hybrid structure in P. mirabilis HN2p), MK861851 (PmGRI1-C55), MF805806 (Tn6450), and CP052444 (pC16KP0098-1). Genes and ORFs are shown as arrows, and arrowheads indicate their transcription orientations. Shared regions with an identity of over $99 \%$ are indicated by shading. Antimicrobial resistance genes are in red, and transposase or integrase genes are in blue. The gray arrows represent other functional genes that have nothing to do with this research. The IS elements are indicated by boxes around the blue arrows. DR represents the 20-bp direct repeats at the ends of PmGRI1-C55. IRi and IRr are the inverted repeats that define the left and right ends of Tn6450, respectively.

At the front end, PmGRI1-HN2p harbors the mobile element IS629 and partial ISEc23 truncated by IS5 and carries a cat A1 resistance gene followed by Tn7-like-HN2p, which might be derived from Tn6450 by partial acquisition according to BLAST analysis (Figure 2a). The acquired part showed high sequence identity to the corresponding regions of four plasmids-pC16KP0098-1 (GenBank accession CP052444), pIncC-L117 (CP040034), pIncC-L121(CP040029), and pIncAC2_L111 (CP030132). The Tn7-like-HN2P region includes three copies of $q n r A 1$ and one copy of $r m t B$. Two of the three copies of qnrA1 were downstream of ISCR1. The duplication of ISCR1 around qnrA1 might facili- 
tate the enhancement of the qnrA1 copy number and accompanying quinolone resistance under conditions of quinolone stress, and it likely adds to the repertoire of mechanisms that can improve quinolone resistance to clinically important levels. The presence of five copies of $q n r A 1$ was also reported in a previous study [45]. The presence of $r m t B$ has been detected in Escherichia coli, Klebsiella pneumoniae, Pseudomonas aeruginosa, Serratia marcescens, and $P$. mirabilis, and the prevalence of such resistance determinants has become concerning [46-51]. The $r m t B$ gene may be located between the Tn2 transposon and insertion element [52,53]. In the Tn7-like-HN2p transposon, $r m t B$ and bla ${ }_{\mathrm{TEM}-1}$ coexist between the Tn2 transposon and ISCR3.

At the back end (Figure 2b), a region similar to PmGRI1-C55 was inverted. An incomplete segment of Tn2 and bla $a_{\mathrm{TEM}-1 \mathrm{~b}}$ is included between the two IS26 and can confer resistance to penicillin and first-generation cephalosporins. IS26 can promote the accumulation of resistance genes on gene islands. Excluding the Tn21 region and five resistance genes (bla $a_{\mathrm{TEM}-1 \mathrm{~b}}, a p h A 1 a, \operatorname{sul2}$, str $A$, and $\left.\operatorname{str} B\right)$, PmGRI1-HN2p carries a mercury-resistance operon. The 18,166-bp region of Tn7-like-HN2p (corresponding to bases 171,395 to 189,560 in MT585156), carrying floR, sul2, aph(4)-Ia, and aacC4 resistance genes, showed high identity to the corresponding regions of seven IncHI2 plasmids-pHNSHP45-2 (GenBank accession KU341381), pHNYJC8 (KY019259), pWJ1 (KY924928), pHNLDF400 (KY019258), pHXY0908 (KM877269), pXGE1mcr (KY990887), and pSJ_255 (CP011062). Similar to previous reports, a class 1 integron was present in Tn7-like-HN2p containing $\operatorname{lun}(\mathrm{F}), d f r A 1$, and aadA1 cassettes [54].

The GI and Tn7 transposons generate new variants through a series of molecular module rearrangements, acquisitions, or losses. The involvement of IS3 and IS21 elements in the rearrangement of multidrug resistance genes on GI has been reported in multidrugresistant E. coli isolates [42]. In Trueperella pyogenes isolated from the lung tissue of cows in Jilin Province, China, multiple drug resistance genes were found to cluster on a $42 \mathrm{~kb}$ genomic island. Two IS6100 1 and a class 1 integron-like SGI1 mediated genetic rearrangements and formed a complex transposon [55]. However, genome rearrangement occurring between the two genetic elements described in this study has seldom been reported. Inversion meditated by transposons and insertion elements found in bacteria might increase, and hybrid structures formed in this way might exist extensively in the natural environment. The effects it will cause and whether it will aggravate the dissemination of antimicrobial resistance genes require further research.

\section{Conclusions}

PmGRI1 is a novel GI that can carry multiple antibiotic resistance genes. The monitoring of PmGRI1 in P. mirabilis and other pathogenic bacteria is currently underway. In this study, we isolated and identified P. mirabilis from a large-scale farm using SS medium and the BD Phoenix ${ }^{\mathrm{TM}} 100$ Automated Microbiology System and screened for multidrug-resistant $P$. mirabilis using the K-B disk diffusion method. We then performed whole-genome sequencing of the screened $P$. mirabilis. This study describes the detailed genetic structure of three novel variants of PmGRI1 and a hybrid structure in which the Tn7-like-HN2p transposon coexists with PmGRI1-HN2p in P. mirabilis. They all carry various important antibiotic resistance genes, which may lead to a more severe spread of antibiotic resistance genes in animals and the environment. This finding highlights the important role of genetic elements (Tn7 and PmGRI1) in capturing and spreading resistance genes in P. mirabilis. Therefore, strong measures are required to control the emergence and spread of antibiotic resistance genes mediated by mobile genetic elements, and our study provides an important reference for this purpose.

Supplementary Materials: The following are available online at https:/ / www.mdpi.com/article/10 .3390/antibiotics10101268/s1, Table S1: Criteria for antibiotic susceptibility of P. mirabilis.

Author Contributions: B.M. wrote the manuscript, X.W. designed the experiments, and B.M., X.W., C.L., Y.T., J.H., Y.G. and Y.Z. performed the experiments and analyzed the data, H.W. provided 
financial support and reviewed the manuscript. All authors contributed to manuscript revision. All authors have read and agreed to the published version of the manuscript.

Funding: The General Program of National Natural Science Foundation of China (No. 32100147 and 31830098); National Key Research and Development Program of China (No. 2018YFD0500305); The National System for Layer Production Technology (No. CARS-40-K14); Sichuan Science and Technology Program (No. 2020NZZJ001); The Fundamental Research Funds for the Central Universities (No. SCU2019D013).

Institutional Review Board Statement: Not applicable.

Informed Consent Statement: Not applicable.

Data Availability Statement: The datasets for this study were obtained from the NCBI MW699442 (PmGRI1-HN31), MW699444 (PmGRI1-YN8), MW699445 (PmGRI1-YN9), CP046048 (P. mirabilisHN2p genome), and MT585156 (hybrid structure).

Conflicts of Interest: The authors declare no conflict of interest.

\section{References}

1. Schaffer, J.N.; Pearson, M.M. Proteus mirabilis and Urinary Tract Infections. Microbiol. Spectr. 2015, 3, 383-433. [CrossRef]

2. Hu, Y.Y.; Cai, J.C.; Zhang, R.; Zhou, H.W.; Sun, Q.; Chen, G.X. Emergence of Proteus mirabilis harboring blaKPC-2 and qnrD in a Chinese Hospital. Antimicrob. Agents Chemother. 2012, 56, 2278-2282. [CrossRef]

3. Ramos, A.C.; Cayô, R.; Carvalhaes, C.G.; Jové, T.; da Silva, G.P.; Sancho, F.M.P.; Chagas-Neto, T.; Medeiros, E.A.S.; Gales, A.C. Dissemination of Multidrug-Resistant Proteus mirabilis Clones Carrying a Novel Integron-Borne bla(IMP-1) in a Tertiary Hospital. Antimicrob. Agents Chemother. 2018, 62, e01321-17. [CrossRef] [PubMed]

4. Sanches, M.S.; Baptista, A.A.S.; de Souza, M.; Menck-Costa, M.F.; Koga, V.L.; Kobayashi, R.K.T.; Rocha, S.P.D. Genotypic and phenotypic profiles of virulence factors and antimicrobial resistance of Proteus mirabilis isolated from chicken carcasses: Potential zoonotic risk. Braz. J. Microbiol. 2019, 50, 685-694. [CrossRef] [PubMed]

5. Li, P.; Liu, D.; Zhang, X.; Tuo, H.; Lei, C.; Xie, X.; Gu, J.; Zhang, A. Characterization of Plasmid-Mediated Quinolone Resistance in Gram-Negative Bacterial Strains from Animals and Humans in China. Microb. Drug Resist. 2019, 25, 1050-1056. [CrossRef]

6. Bushen, A.; Tekalign, E.; Abayneh, M. Drug- and Multidrug-Resistance Pattern of Enterobacteriaceae Isolated from Droppings of Healthy Chickens on a Poultry Farm in Southwest Ethiopia. Infect. Drug Resist. 2021, 14, 2051-2058. [CrossRef]

7. Lei, C.W.; Chen, Y.P.; Kong, L.H.; Zeng, J.X.; Wang, Y.X.; Zhang, A.Y.; Wang, H.N. PGI2 Is a Novel SGI1-Relative MultidrugResistant Genomic Island Characterized in Proteus mirabilis. Antimicrob. Agents Chemother. 2018, 62, e00019-18. [CrossRef] [PubMed]

8. Wang, X.C.; Lei, C.W.; Kang, Z.Z.; Zhang, Y.; Wang, H.N. IS26-Mediated Genetic Rearrangements in Salmonella Genomic Island 1 of Proteus mirabilis. Front. Microbiol. 2019, 10, 2245. [CrossRef] [PubMed]

9. Lei, C.W.; Zhang, A.Y.; Liu, B.H.; Wang, H.N.; Yang, L.Q.; Guan, Z.B.; Xu, C.W.; Zhang, D.D.; Yang, Y.Q. Two novel Salmonella genomic island 1 variants in Proteus mirabilis isolates from swine farms in China. Antimicrob. Agents Chemother. 2015, 59, 4336-4338. [CrossRef] [PubMed]

10. Chen, Y.P.; Lei, C.W.; Kong, L.H.; Zeng, J.X.; Zhang, X.Z.; Liu, B.H.; Li, Y.; Xiang, R.; Wang, Y.X.; Chen, D.Y.; et al. Tn6450, a Novel Multidrug Resistance Transposon Characterized in a Proteus mirabilis Isolate from Chicken in China. Antimicrob. Agents Chemother. 2018, 62, e02192-17. [CrossRef] [PubMed]

11. Delavat, F.; Miyazaki, R.; Carraro, N.; Pradervand, N.; van der Meer, J.R. The hidden life of integrative and conjugative elements. FEMS Microbiol. Rev. 2017, 41, 512-537. [CrossRef]

12. Partridge, S.R.; Kwong, S.M.; Firth, N.; Jensen, S.O. Mobile Genetic Elements Associated with Antimicrobial Resistance. Clin. Microbiol. Rev. 2018, 31, e00088-17. [CrossRef] [PubMed]

13. Bellanger, X.; Payot, S.; Leblond-Bourget, N.; Guédon, G. Conjugative and mobilizable genomic islands in bacteria: Evolution and diversity. FEMS Microbiol. Rev. 2014, 38, 720-760. [CrossRef]

14. Liu, D.; Liu, W.; Lv, Z.; Xia, J.; Li, X.; Hao, Y.; Zhou, Y.; Yao, H.; Liu, Z.; Wang, Y.; et al. Emerging erm(B)-Mediated Macrolide Resistance Associated with Novel Multidrug Resistance Genomic Islands in Campylobacter. Antimicrob. Agents Chemother. 2019, 63, e00153-19. [CrossRef]

15. Yao, H.; Jiao, D.; Zhao, W.; Li, A.; Li, R.; Du, X.D. Emergence of a Novel tet(L) Variant in Campylobacter spp. of Chicken Origin in China. Antimicrob. Agents Chemother. 2020, 65, e01622-20. [CrossRef]

16. Zhu, D.; Yang, Z.; Xu, J.; Wang, M.; Jia, R.; Chen, S.; Liu, M.; Zhao, X.; Yang, Q.; Wu, Y.; et al. Pan-genome analysis of Riemerella anatipestifer reveals its genomic diversity and acquired antibiotic resistance associated with genomic islands. Funct. Integr. Genomics 2020, 20, 307-320. [CrossRef] [PubMed]

17. Dong, W.L.; Xu, Q.J.; Atiah, L.A.; Odah, K.A.; Gao, Y.H.; Kong, L.C.; Ma, H.X. Genomic island type IV secretion system and transposons in genomic islands involved in antimicrobial resistance in Trueperella pyogenes. Vet. Microbiol. 2020, $242,108602$. [CrossRef] [PubMed] 
18. Ahmed, A.M.; Hussein, A.I.; Shimamoto, T. Proteus mirabilis clinical isolate harbouring a new variant of Salmonella genomic island 1 containing the multiple antibiotic resistance region. J. Antimicrob. Chemother. 2007, 59, 184-190. [CrossRef] [PubMed]

19. Doublet, B.; Poirel, L.; Praud, K.; Nordmann, P.; Cloeckaert, A. European clinical isolate of Proteus mirabilis harbouring the Salmonella genomic island 1 variant SGI1-O. J. Antimicrob. Chemother. 2010, 65, 2260-2262. [CrossRef]

20. Siebor, E.; Neuwirth, C. Emergence of Salmonella genomic island 1 (SGI1) among Proteus mirabilis clinical isolates in Dijon, France. J. Antimicrob. Chemother. 2013, 68, 1750-1756. [CrossRef]

21. Siebor, E.; Neuwirth, C. Proteus genomic island 1 (PGI1), a new resistance genomic island from two Proteus mirabilis French clinical isolates. J. Antimicrob. Chemother. 2014, 69, 3216-3220. [CrossRef] [PubMed]

22. Girlich, D.; Dortet, L.; Poirel, L.; Nordmann, P. Integration of the blaNDM-1 carbapenemase gene into Proteus genomic island 1 (PGI1-PmPEL) in a Proteus mirabilis clinical isolate. J. Antimicrob. Chemother. 2015, 70, 98-102. [CrossRef]

23. Cummins, M.L.; Roy Chowdhury, P.; Marenda, M.S.; Browning, G.F.; Djordjevic, S.P. Salmonella Genomic Island 1B Variant Found in a Sequence Type 117 Avian Pathogenic Escherichia coli Isolate. mSphere 2019, 4, e00169-19. [CrossRef]

24. Schultz, E.; Barraud, O.; Madec, J.Y.; Haenni, M.; Cloeckaert, A.; Ploy, M.C.; Doublet, B. Multidrug Resistance Salmonella Genomic Island 1 in a Morganella morganii subsp. morganii Human Clinical Isolate from France. mSphere 2017, 2, e00118-17. [CrossRef]

25. Soliman, A.M.; Shimamoto, T.; Nariya, H.; Shimamoto, T. Emergence of Salmonella Genomic Island 1 Variant SGI1-W in a Clinical Isolate of Providencia stuartii from Egypt. Antimicrob. Agents Chemother. 2019, 63, e01793-18. [CrossRef] [PubMed]

26. Siebor, E.; de Curraize, C.; Neuwirth, C. Genomic context of resistance genes within a French clinical MDR Proteus mirabilis: Identification of the novel genomic resistance island GIPmi1. J. Antimicrob. Chemother. 2018, 73, 1808-1811. [CrossRef] [PubMed]

27. Lei, C.W.; Yao, T.G.; Yan, J.; Li, B.Y.; Wang, X.C.; Zhang, Y.; Gao, Y.F.; Wang, H.N. Identification of Proteus genomic island 2 variants in two clonal Proteus mirabilis isolates with coexistence of a novel genomic resistance island PmGRI1. J. Antimicrob. Chemother. 2020, 75, 2503-2507. [CrossRef] [PubMed]

28. Chen, Y.; Lei, C.; Zuo, L.; Kong, L.; Kang, Z.; Zeng, J.; Zhang, X.; Wang, H. A novel cfr-carrying Tn7 transposon derivative characterized in Morganella morganii of swine origin in China. J. Antimicrob. Chemother. 2019, 74, 603-606. [CrossRef]

29. Peters, J.E.; Craig, N.L. Tn7: Smarter than we thought. Nat. Rev. Mol. Cell Biol. 2001, 2, 806-814. [CrossRef]

30. Li, H.; Durbin, R. Fast and accurate long-read alignment with Burrows-Wheeler transform. Bioinformatics 2010, 26, 589-595. [CrossRef]

31. McKenna, A.; Hanna, M.; Banks, E.; Sivachenko, A.; Cibulskis, K.; Kernytsky, A.; Garimella, K.; Altshuler, D.; Gabriel, S.; Daly, M.; et al. The Genome Analysis Toolkit: A MapReduce framework for analyzing next-generation DNA sequencing data. Genome Res. 2010, 20, 1297-1303. [CrossRef] [PubMed]

32. Cingolani, P.; Platts, A.; Wang le, L.; Coon, M.; Nguyen, T.; Wang, L.; Land, S.J.; Lu, X.; Ruden, D.M. A program for annotating and predicting the effects of single nucleotide polymorphisms, SnpEff: SNPs in the genome of Drosophila melanogaster strain w1118; iso-2; iso-3. Fly (Austin) 2012, 6, 80-92. [CrossRef]

33. Abyzov, A.; Urban, A.E.; Snyder, M.; Gerstein, M. CNVnator: An approach to discover, genotype, and characterize typical and atypical CNVs from family and population genome sequencing. Genome Res. 2011, 21, 974-984. [CrossRef]

34. Wang, J.; Mullighan, C.G.; Easton, J.; Roberts, S.; Heatley, S.L.; Ma, J.; Rusch, M.C.; Chen, K.; Harris, C.C.; Ding, L.; et al. CREST maps somatic structural variation in cancer genomes with base-pair resolution. Nat. Methods 2011, 8, 652-654. [CrossRef] [PubMed]

35. Koren, S.; Walenz, B.P.; Berlin, K.; Miller, J.R.; Bergman, N.H.; Phillippy, A.M. Canu: Scalable and accurate long-read assembly via adaptive k-mer weighting and repeat separation. Genome Res. 2017, 27, 722-736. [CrossRef]

36. Zankari, E.; Hasman, H.; Cosentino, S.; Vestergaard, M.; Rasmussen, S.; Lund, O.; Aarestrup, F.M.; Larsen, M.V. Identification of acquired antimicrobial resistance genes. J. Antimicrob. Chemother. 2012, 67, 2640-2644. [CrossRef]

37. Siguier, P.; Perochon, J.; Lestrade, L.; Mahillon, J.; Chandler, M. ISfinder: The reference centre for bacterial insertion sequences. Nucleic Acids Res. 2006, 34, D32-D36. [CrossRef]

38. Carattoli, A.; Zankari, E.; García-Fernández, A.; Voldby Larsen, M.; Lund, O.; Villa, L.; Møller Aarestrup, F.; Hasman, H. In silico detection and typing of plasmids using PlasmidFinder and plasmid multilocus sequence typing. Antimicrob. Agents Chemother. 2014, 58, 3895-3903. [CrossRef]

39. Clausen, P.; Aarestrup, F.M.; Lund, O. Rapid and precise alignment of raw reads against redundant databases with KMA. BMC Bioinform. 2018, 19, 307. [CrossRef] [PubMed]

40. Blackwell, G.A.; Nigro, S.J.; Hall, R.M. Evolution of AbGRI2-0, the Progenitor of the AbGRI2 Resistance Island in Global Clone 2 of Acinetobacter baumannii. Antimicrob. Agents Chemother. 2015, 60, 1421-1429. [CrossRef]

41. He, S.; Hickman, A.B.; Varani, A.M.; Siguier, P.; Chandler, M.; Dekker, J.P.; Dyda, F. Insertion Sequence IS26 Reorganizes Plasmids in Clinically Isolated Multidrug-Resistant Bacteria by Replicative Transposition. mBio 2015, 6, e00762. [CrossRef]

42. Proença, J.T.; Barral, D.C.; Gordo, I. Commensal-to-pathogen transition: One-single transposon insertion results in two pathoadaptive traits in Escherichia coli-macrophage interaction. Sci. Rep. 2017, 7, 4504. [CrossRef]

43. Harmer, C.J.; Hall, R.M. IS26-Mediated Formation of Transposons Carrying Antibiotic Resistance Genes. mSphere 2016, 1, e00038-16. [CrossRef]

44. Harmer, C.J.; Hall, R.M. IS26-Mediated Precise Excision of the IS26-aphA1a Translocatable Unit. mBio 2015, 6, e01866-15. [CrossRef] [PubMed] 
45. Vinué, L.; Sater, M.R.A.; Herriott, I.; Huntley, M.H.; Jacoby, G.A.; Hooper, D.C. Multiple Copies of qnrA1 on an IncA/C(2) Plasmid Explain Enhanced Quinolone Resistance in an Escherichia coli Mutant. Antimicrob. Agents Chemother. 2019, 63, e00718-19. [CrossRef]

46. Fritsche, T.R.; Castanheira, M.; Miller, G.H.; Jones, R.N.; Armstrong, E.S. Detection of methyltransferases conferring high-level resistance to aminoglycosides in enterobacteriaceae from Europe, North America, and Latin America. Antimicrob. Agents Chemother. 2008, 52, 1843-1845. [CrossRef] [PubMed]

47. Cassu-Corsi, D.; Martins, W.M.; Nicoletti, A.G.; Almeida, L.G.; Vasconcelos, A.T.; Gales, A.C. Characterisation of plasmidmediated rmtB-1 in Enterobacteriaceae clinical isolates from São Paulo, Brazil. Memórias do Instituto Oswaldo Cruz 2018, 113, e180392. [CrossRef] [PubMed]

48. Yokoyama, K.; Doi, Y.; Yamane, K.; Kurokawa, H.; Shibata, N.; Shibayama, K.; Yagi, T.; Kato, H.; Arakawa, Y. Acquisition of 16S rRNA methylase gene in Pseudomonas aeruginosa. Lancet 2003, 362, 1888-1893. [CrossRef]

49. Yamane, K.; Wachino, J.; Doi, Y.; Kurokawa, H.; Arakawa, Y. Global spread of multiple aminoglycoside resistance genes. Emerg. Infect. Dis. 2005, 11, 951-953. [CrossRef]

50. Doi, Y.; Yokoyama, K.; Yamane, K.; Wachino, J.; Shibata, N.; Yagi, T.; Shibayama, K.; Kato, H.; Arakawa, Y. Plasmid-mediated 16S rRNA methylase in Serratia marcescens conferring high-level resistance to aminoglycosides. Antimicrob. Agents Chemother. 2004, 48, 491-496. [CrossRef] [PubMed]

51. Wachino, J.; Yamane, K.; Shibayama, K.; Kurokawa, H.; Shibata, N.; Suzuki, S.; Doi, Y.; Kimura, K.; Ike, Y.; Arakawa, Y. Novel plasmid-mediated 16S rRNA methylase, $\mathrm{RmtC}$, found in a Proteus mirabilis isolate demonstrating extraordinary high-level resistance against various aminoglycosides. Antimicrob. Agents Chemother. 2006, 50, 178-184. [CrossRef] [PubMed]

52. Du, X.D.; Wu, C.M.; Liu, H.B.; Li, X.S.; Beier, R.C.; Xiao, F.; Qin, S.S.; Huang, S.Y.; Shen, J.Z. Plasmid-mediated ArmA and RmtB 16S rRNA methylases in Escherichia coli isolated from chickens. J. Antimicrob. Chemother. 2009, 64, 1328-1330. [CrossRef]

53. Doi, Y.; Adams-Haduch, J.M.; Paterson, D.L. Escherichia coli isolate coproducing $16 \mathrm{~S}$ rRNA Methylase and CTX-M-type extendedspectrum beta-lactamase isolated from an outpatient in the United States. Antimicrob. Agents Chemother. 2008, 52, 1204-1205. [CrossRef] [PubMed]

54. Siebor, E.; Neuwirth, C. The new variant of Salmonella genomic island 1 (SGI1-V) from a Proteus mirabilis French clinical isolate harbours blaVEB-6 and qnrA1 in the multiple antibiotic resistance region. J. Antimicrob. Chemother. 2011, 66, 2513-2520. [CrossRef]

55. Dong, W.L.; Odah, K.A.; Liu, L.; Xu, Q.J.; Gao, Y.H.; Kong, L.C.; Ma, H.X. Multidrug resistance genes are associated with a 42-kb island TGI1 carrying a complex class 1 integron in Trueperella pyogenes. J. Glob. Antimicrob. Resist. 2020, 22, 1-4. [CrossRef] [PubMed] 